\title{
Thermo-Statistics of Irreversible Processes: a Boltzmann-Gibbs-Style Ensemble Formalism
}

\author{
Roberto Luzzi, Áurea R. Vasconcellos, and J. Galvão Ramos \\ Instituto de Física 'Gleb Wataghin', Universidade Estadual de Campinas, Unicamp, 13083-970, Campinas, SP, Brazil
}

Received on 19 July, 2005; accepted on 28 November, 2005

\begin{abstract}
The area of Physics indicated in the title is nowadays of quite relevant interest, not only from the purely scientific point of view, but specially for its applied aspects associated to the present-time point-first-technologies. A particular research trend in the theory of irreversible processes, which are evolving in time in systems arbitrarily departed from equilibrium, is here briefly described. It consists in the construction of a Gibbs-style nonequilibrium ensemble formalism. The derivation of a nonequilibrium statistical operator is described (the variational approach of Predictive Statistical Mechanics is used). The main questions involved are presented and applications are briefly mentioned.
\end{abstract}

Keywords: Thermostatistics; Irreversible processes; Nonequilibrium statistics

\section{INTRODUCTION}

It is generally considered that the aim of Statistical Mechanics of many-body systems away from equilibrium is to determine their thermodynamic properties, and the evolution in time of their macroscopic observables, in terms of the dynamical laws which govern the motion of their constitutive elements. This implies, first, in the construction of an irreversible thermodynamics and a thermo-hydrodynamics (the latter meaning the particle and energy motion in fluids, rheological properties, etc., with the transport coefficients depending on the macroscopic thermodynamic state of the system). Second, we need to face the all-important derivation of a generalized nonlinear quantum kinetic theory and a response function theory, which are of fundamental relevance to connect theory with observation and experiment, basic for the corroboration of any theory [1], that is, the synthesis leg in the scientific method born in the seventeenth century.

Oliver Penrose [2] has noted that Statistical Mechanics is notorious for conceptual problems to which is difficult to give a convincing answer, mainly:

What is the physical significance of a Gibbs' ensemble?;

How can we justify the standard ensembles used in equilibrium theory?;

What are the right ensembles for nonequilibrium problems?;

How can we reconcile the reversibility of microscopic mechanics with the irreversibility of macroscopic behavior?

Moreover, related to the case of many-body systems out of equilibrium, the late Ryogo Kubo, in the opening address in the Oji Seminar [3], told us that statistical mechanics of nonlinear nonequilibrium phenomena is just in its infancy and further progress can only be hoped by closed cooperation with experiment. Some progress has been achieved since then, and we try in this review to describe, in a simple manner, some attempts in the direction to provide a path for one particular initial programme to face the questions posited above.

Statistical Mechanics is a grandiose theoretical construction whose founding fathers include the great names of James C. Maxwell, Ludwig Boltzmann and J. Willard Gibbs [4].
We may recall that it is fundamental for the study of condensed matter, which could be said to be statistical mechanics by antonomasia. Therefore statistical mechanics can be considered the science mother of the present day advanced technology, which is the base of our sophisticated contemporary civilization. Its application to the case of systems in equilibrium proceeded rapidly and with exceptional success: equilibrium statistical mechanics gave - starting from the microscopic level - foundations to Thermostatics, its original objective, and the possibility to build a Response Function Theory. Applications to nonequilibrium systems began, mainly, with the case of local equilibrium in the linear regime following the pioneering work of Lars Onsager (see, for example, [5]).

For systems arbitrarily deviated from equilibrium and governed by nonlinear kinetic laws, the derivation of an ensemble-like formalism proceeded at a slower pace than in the case of equilibrium, and somewhat cautiously. A long list of distinguished scientists contributed to such development, and among them we can mention Nicolai Bogoliubov, John Kirkwood, Sergei Krylov, Melvin Green, Robert Zwanzig, Hazimi Mori, Ilya Prigogine, Dimitri Zubarev. It must be added the name of Edwin Jaynes, who systematized, or better to say codified, the matter on the basis of a variational principle in the context of what is referred to as Predictive Statistical Mechanics [6-13], which is based on a framework provided by Information Theory.

It can be noticed that the subject involves a number of questions to which it is necessary to give an answer, namely

1. The question of the choice of the basic variables

2. The question of irreversibility

3. The question of the initial value condition

4. The question of historicity

5. The question of providing the statistical operator

6. The question of building a non-equilibrium grandcanonical ensemble

7. The question of the truncation procedure 
8. The question of the equations of evolution (nonlinear quantum kinetic theory)

9. The question of a response function theory

10. The question of validation (experiment and theory)

11. The question of the approach to equilibrium

12. The question of a non-equilibrium statistical thermodynamics

13. The question of a thermo-statistical approach to complex systems

14. The question of a nonlinear higher-order thermohydrodynamics

15. The question of statistical mechanics for complex structured systems

which are addressed in $[13,14]$.

In the study of the macroscopic state of nonequilibrium systems we face greater difficulties than those present in the theory of equilibrium systems. This is mainly due to the fact that a more detailed analysis is necessary to determine the temporal dependence of measurable properties, and to calculate transport coefficients which are time-dependent (that is, depending on the evolution in time of the nonequilibrium macrostate of the system where dissipative processes are unfolding), and which are also space dependent. That dependence is nonlocal in space and non-instantaneous in time, as it encompasses space and time correlations. Robert Zwanzig [15] has summarized the basic goals of nonequilibrium statistical mechanics as consisting of: (i) To derive transport equations and to grasp their structure; (ii) To understand how the approach to equilibrium occurs in natural isolated systems; (iii) To study the properties of steady states; and (iv) To calculate the instantaneous values and the temporal evolution of the physical quantities which specify the macroscopic state of the system. Also according to Zwanzig, for the purpose to face these items, there exist several approaches which can be classified as: (a) Intuitive techniques; (b) Techniques based on the generalization of the theory of gases; (c) Techniques based on the theory of stochastic processes; (d) Expansions from an initial equilibrium ensemble; (e) Generalization of Gibbs' ensemble formalism.

The last item (e) is connected with Penrose's questions noticed above concerning if there are, and what are, right ensembles for nonequilibrium problems. In the absence of a Gibbsstyle ensemble approach, for a long time different kinetic theories were used, with variable success, to deal with the great variety of nonequilibrium phenomena occurring in physical systems in nature. We describe here a proposition for the construction of a Nonequilibrium Statistical Ensemble Formalism, or NESEF, for short, which appears to provide grounds for a general prescription to choose appropriate ensembles for nonequilibrium systems. The formalism has an accompanying nonlinear quantum transport theory of a large scope (which encompasses as particular limiting cases Boltzmann's and
Mori's approaches), a response function theory for arbitrarilyaway-from-equilibrium systems, a statistical thermodynamics (the so-called Informational Statistical Thermodynamics), and an accompanying Thermo-Hydrodynamics.

NESEF appears as a very powerful, concise, based on sound principles, and elegant formalism of a broad scope to deal with systems arbitrarily away from equilibrium. Zwanzig stated that the formalism "has by far the most appealing structure, and may yet become the most effective method for dealing with nonlinear transport processes" [15]. Later developments have confirmed Zwanzig's prediction. The present structure of the formalism consists in a vast extension and generalization of earlier pioneering approaches, among which we can pinpoint the works of Kirkwood [16], Green [17], MoriOppenheim-Ross [18], Mori [19], and Zwanzig [20]. NESEF has been approached from different points of view: some are based on heuristic arguments [18, 21-24], others on projection operator techniques [25-27] (the former following Kirkwood and Green and the latter following Zwanzig and Mori). The formalism has been particularly systematized and largely improved by the Russian School of statistical physics, which can be considered to have been initiated by the renowned Nicolai Nicolaievich Bogoliubov [28], and we may also name Nicolai Sergeivich Krylov [29], and more recently mainly through the relevant contributions by Dimitrii Zubarev [24, 30], Sergei Peletminskii [22, 23], and others.

These different approaches to NESEF can be brought together under a unique variational principle. This has been originally done by Zubarev and Kalashnikov [31], and later on reconsidered in Ref. [32] (see also Refs. [33] and [34]). It consists on the maximization, in the context of Information Theory, of Gibbs statistical entropy (to be called fine-grained informational-statistical entropy), subjected to certain constraints, and including non-locality in space, retro-effects, and irreversibility on the macroscopic level. This is the foundation of the nonequilibrium statistical ensemble formalism that we describe in general terms in following sections. The topic has surfaced in the section "Questions and Answers" of the Am. J. Phys. [6, 35]. The question by Baierlein [35], "A central organizing principle for statistical and thermal physics?", was followed by Semura's answer [6] that "the best central organizing principle for statistical and thermal physics is that of maximum [informational] entropy [...]. The principle states that the probability should be chosen to maximize the average missing information of the system, subjected to the constraints imposed by the [available] information. This assignment is consistent with the least biased estimation of probabilities."

The formalism may be considered as covered under the umbrella provided by the scheme of Jaynes' Predictive Statistical Mechanics [7]. This is a powerful approach based on the Bayesian method in probability theory, together with the principle of maximization of informational entropy (MaxEnt), and the resulting statistical ensemble formalism is referredto as MaxEnt-NESEF. Jaynes' scheme implies in a predictive statistics that is built only on the access to the relevant information that there exists of the system [6-12]. As pointed out by Jaynes [8]. "How shall we best think about Nature and most efficiently predict her behavior, given only our incom- 
plete knowledge [of the microscopic details of the system]? [...]. We need to see it, not as an example of the N-body equations of motion, but as an example of the logic of scientific inference, which by-passes all details by going directly from our macroscopic information to the best macroscopic predictions that can be made from that information" (emphasis is ours) [...]. "Predictive Statistical Mechanics is not a physical theory, but a method of reasoning that accomplishes this by finding, not the particular that the equations of motion say in any particular case, but the general things that they say in 'almost all' cases consisting with our information; for those are the reproducible things".

Again following Jaynes' reasoning, the construction of a statistical approach is based on "a rather basic principle [...]: If any macrophenomenon is found to be reproducible, then it follows that all microscopic details that were not under the experimenters' control must be irrelevant for understanding and predicting it". Further, "the difficulty of prediction from microstates lies [..] in our own lack of the information needed to apply them. We never know the microstates; only a few aspects of the macrostate. Nevertheless, the aforementioned principle of [macroscopic] reproducibility convinces us that this should be enough; the relevant information is there, if only we can see how to recognize it and use it" [emphasis is ours].

As noticed, Predictive Statistical Mechanics is founded on the Bayesian approach in probability theory. According to Jaynes, the question of what are theoretically valid, and pragmatically useful, ways of applying probability theory in science has been approached by Sir Harold Jeffreys [36, 37], in the sense that he stated the general philosophy of what scientific inference is and proceeded to carry both the mathematical theory and its implementations. Together with Jaynes and others, the Nobelist Philip W. Anderson [38] maintains that what seems to be the most appropriate probability theory for the sciences is the Bayesian approach. The Bayesian interpretation is that probability is the degree of belief which is consistent to hold in considering a proposition as being true, once other conditioning propositions are taken as true [39]. Or, also according to Anderson: "What Bayesian does is to focus one's attention on the question one wants to ask of the data. It says in effect, how do these data affect my previous knowledge of the situation? It is sometimes called maximum likelihood thinking, but the essence of it is to clearly identify the possible answers, assign reasonable a priori probabilities to them and then ask which answers have been done more likely by the data" [emphasis is ours].

The question that arises is, as stated by Jaynes, "how shall we use probability theory to help us do plausible reasoning in situations where, because of incomplete information we cannot use deductive reasoning?" In other words, the main question is how to obtain the probability assignment compatible with the available information, while avoiding unwarranted assumptions. This is answered by Jaynes who formulated the criterion that: the least biased probability assignment $\left\{p_{j}\right\}$, for a set of mutually exclusive events $\left\{x_{j}\right\}$, is the one that maximizes the quantity $S_{I}$, sometimes referred to as the infor- mational entropy, given by

$$
S_{I}=-\sum_{j} p_{j} \ln p_{j}
$$

conditioned by the constraints imposed by the available information. This is based on Shannon's ideas in the mathematical theory of communications [40], who first demonstrated that, for an exhaustive set of mutually exclusive propositions, there exists a unique function measuring the uncertainty of the probability assignment. This is the already mentioned principle of maximization of the informational-statistical entropy, MaxEnt for short. It provides the variational principle which results in a unifying theoretical framework for NESEF, thus introducing, as we have noticed, MaxEnt-NESEF as a nonequilibrium statistical ensemble formalism. It should be stressed that the maximization of $S_{I}$ implies in making maximum the uncertainty in the information available (in Shannon-Brillouin's sense $[40,41])$, to have in fact the least biased probability assignment.

We proceed next to describe the construction of NESEF and of an irreversible thermodynamics founded on its premises. This is done, as indicated above, in the context of the variational principle MaxEnt, but an alternative derivation along traditional (heuristic) ways is also possible and described in Ref. [14].

\section{A NONEQUILIBRIUM STATISTICAL ENSEMBLE FORMALISM}

In the construction of nonequilibrium statistical ensembles, that is, a Nonequilibrium Statistical Ensemble Formalism (NESEF), basically consisting into the derivation of a nonequilibrium statistical operator (probability distribution in the classical case), first it needs be noticed that for systems away from equilibrium several important points need be carefully taken into account in each case under consideration [cf. the list of questions above], particularly:

(1) The choice of the basic variables (a wholly different choice than in equilibrium when it suffices to take a subset of those which are constants of motion), which is to be based on an analysis of what sort of macroscopic measurements and processes are actually possible, and, moreover one is to focus attention not only on what can be observed but also on the character and expectative concerning the equations of evolution for these variables (e.g. Refs. [15, 42]). We also notice that even though at the initial stage we would need to introduce all the observables of the system, as time elapses more and more contracted descriptions can be used as enters into play Bogoliubov's principle of correlation weakening and the accompanying hierarchy of relaxation times [42].

It can be noticed that to consider all the observables of the system is consisting with introducing the reduced oneparticle, $\hat{n}_{1}$, and two-particle, $\hat{n}_{2}$, dynamical operators [13, $14,42,43]$ in classical mechanics given by

$$
\hat{n}_{1}(\mathbf{r}, \mathbf{p})=\sum_{j} \delta\left(\mathbf{r}-\mathbf{r}_{j}\right) \boldsymbol{\delta}\left(\mathbf{p}-\mathbf{p}_{j}\right)
$$




$$
\hat{n}_{2}\left(\mathbf{r}, \mathbf{p} ; \mathbf{r}^{\prime}, \mathbf{p}^{\prime}\right)=\sum_{j \neq k} \delta\left(\mathbf{r}-\mathbf{r}_{j}\right) \delta\left(\mathbf{p}-\mathbf{p}_{j}\right) \delta\left(\mathbf{r}^{\prime}-\mathbf{r}_{k}\right) \delta\left(\mathbf{p}^{\prime}-\mathbf{p}_{k}\right)
$$

with $\mathbf{r}_{j}$ and $\mathbf{p}_{j}$ being the coordinate and linear momentum of the $j$-th particle in phase space and $\mathbf{r}$ and $\mathbf{p}$ the continuous values of position and momentum, which are called field variables (for the quantum case see [13]). For simplicity we are considering a system of $N$ particles of mass $m$; the case of systems with several kinds of particles are straightforwardly included in the treatment: it suffices to introduce a second index to indicate them, i.e $\mathbf{r}_{s j}, \mathbf{p}_{s j}$, etc. [see Subsection $\mathbf{4 . 2}$ below].

But it is pertinent to look for what can be termed as a generalized grand-canonical ensemble, what can be done [13] by introducing in place of $\hat{n}_{1}$, and $\hat{n}_{2}$ independent linear combination of them. For simplicity consider only $\hat{n}_{1}$, and the new variables are the densities of kinetic energy and of particles

$$
\hat{h}(\mathbf{r})=\int d^{3} p \frac{p^{2}}{2 m} \hat{n}_{1}(\mathbf{r}, \mathbf{p}) \quad ; \quad \hat{n}(\mathbf{r})=\int d^{3} p \hat{n}_{1}(\mathbf{r}, \mathbf{p})
$$

and their fluxes of all order, namely,

$$
\begin{gathered}
\hat{I}_{h}^{[r]}(\mathbf{r})=\int d^{3} p u^{[r]}(\mathbf{p}) \frac{p^{2}}{2 m} \hat{n}_{1}(\mathbf{r}, \mathbf{p}) \\
\hat{I}_{n}^{[r]}(\mathbf{r})=\int d^{3} p u^{[r]}(\mathbf{p}) \hat{n}_{1}(\mathbf{r}, \mathbf{p})
\end{gathered}
$$

where $r=1$ for the vectorial flux or current, $r \geq 2$ for the other higher-order fluxes; $r$ also indicates the tensorial rank, and

$$
u^{[r]}(\mathbf{p})=\left[\frac{\mathbf{p}}{m} \ldots(r-\text { times }) \ldots \frac{\mathbf{p}}{m}\right]
$$

stands for the tensorial product of $r$-times the vector $\mathbf{p} / \mathrm{m}$, rendering a tensor of rank $r$. The contributions associated to $\hat{n}_{2}$ are of the form [13]

$$
\hat{C}_{p p^{\prime}}^{[r+r]}\left(\mathbf{r}, \hat{\mathbf{r}}^{\hat{\prime}}\right)=\left[\hat{I}_{p}^{[r]}(\mathbf{r}) \hat{I}_{p^{\prime}}^{\left[r^{\prime}\right]}\left(\mathbf{r}^{\hat{\prime}}\right)\right]
$$

where $p$ and $p^{\prime}$ are indexes $h$ or $n ; r, \quad r=0$ (the densities), $1,2, \ldots$, and $[\ldots]$ as above stands for tensorial product. The question of truncation of description, that is to take a reduced number of the above variables (associated to Bogoliubov's principle of correlation weakening and hierarchy of relaxation times) and the question of the approach to equilibrium is discussed elsewhere [13, 14] (In equilibrium, because there survive only the variables of Eqs. (8) only for $r, r^{\prime}=0$, there follows a nonextensive description, becoming approximately extensive in the thermodynamic limit [14]).

(2) It needs be introduced historicity, that is, the idea that it must be incorporated all the past dynamics of the system (or historicity effects), all along the time interval going from a starting description of the macrostate of the sample in the given experiment, say at $t_{o}$, up to the time $\mathrm{t}$ when a measurement is performed. This is a quite important point in the case of dissipative systems as emphasized among others by John Kirkwood and Hazime Mori. It implies in that the history of the system is not merely the series of events in which the system has been involved, but it is the series of transformations along time by which the system progressively comes into being at time $\mathrm{t}$ (when a measurement is performed), through the evolution governed by the laws of mechanics $[16,18]$.

(3) The question of irreversibility (or Eddington's arrow of time) on what Rudolf Peierls stated that: "In any theoretical treatment of transport problems, it is important to realize at what point the irreversibility has been incorporated. If it has not been incorporated, the treatment is wrong. A description of the situation that preserves the reversibility in time is bound to give the answer zero or infinity for any conductivity. If we do not see clearly where the irreversibility is introduced, we do not clearly understand what we are doing" [44].

The question is then to find the proper nonequilibrium statistical operator that MaxEnt-NESEF should provide. The way out of the difficulties pointed out above is contained in the idea set forward by John Kirkwood in the decade of the ,forties [16]. He pointed out that the state of the system at time $t$ is strongly dependent on all the previous evolution of the nonequilibrium processes that have been developing in it. Kirkwood introduces this fact, in the context of the transport theory he proposes, in the form of a so-called time-smoothing procedure, which is generalized in MaxEnt-NESEF as shown below.

After the choice of the basic dynamical variables has been performed, and let us call them generically as $\left\{\hat{P}_{j}(\xi)\right\}$, where $\xi$ indicates the set of all variables on which the $\hat{P}_{j}$ may depend [cf. Eqs. (2) and (3), and Eqs. (4) to (6) and (8)], introducing in MaxEnt-NESEF [13, 14, 31-34] the idea that it must be incorporated all the past history of the system (or historicity effects), all along the time interval going from the initial condition of preparation of the sample in the given experiment at, say, time $t_{o}$ up to time $t$ when a measurement is performed (i.e., when we observe the macroscopic state of the system), we proceed to maximize Gibbs' entropy (sometimes called fine-grained entropy)

$$
S_{G}(t)=-\operatorname{Tr}\{\rho(t) \ln \rho(t)\} \quad,
$$

with the normalization and constraints given at any time $t^{\prime}$ in the interval $t_{o} \leq t^{\prime} \leq t$, namely

$$
\begin{gathered}
\operatorname{Tr}\left\{\rho\left(t^{\prime}\right)\right\}=1, \\
Q_{j}\left(\xi, t^{\prime}\right)=\operatorname{Tr}\left\{\hat{P}_{j}(\xi) \rho\left(t^{\prime}\right)\right\},
\end{gathered}
$$

with $Q_{j}\left(\xi, t^{\prime}\right)$ being the nonequilibrium thermodynamic (macroscopic) variables for the description of the accompanying irreversible thermodynamics described in next section.

Resorting to Lagrange's procedure we find that

$$
\rho(t)=\exp \left\{-\Psi(t)-\sum_{j} \int d \xi \int_{t_{o}}^{t} d t^{\prime} \varphi_{j}\left(\xi ; t, t^{\prime}\right) \hat{P}_{j}\left(\xi ; t-t^{\prime}\right)\right\},
$$


where

$\Psi(t)=\ln \operatorname{Tr}\left\{\exp \left[-\sum_{j} \int d \xi \int_{t_{o}}^{t} d t^{\prime} \varphi_{j}\left(\xi ; t, t^{\prime}\right) \hat{P}_{j}\left(\xi ; t-t^{\prime}\right)\right]\right\}$,

and the $\varphi_{j}$ are the corresponding Lagrange multipliers determined in terms of the basic macrovariables by Eq. (11), and operators $\hat{P}_{j}$ are given in Heisenberg representation.

Further an additional basic step needs now be considered, namely a generalization of Kirkwood's time-smoothing procedure. This is done introducing an extra assumption on the form of the Lagrange multipliers $\varphi_{j}$, in such a way, we stress, that (i) irreversible behavior in the evolution of the macroscopic state of the system is satisfied; (ii) the instantaneous state of the system is given by Eq. (11); (iii) it is introduced the set of quantities $\left\{F_{j}(\xi, t)\right\}$ as intensive variables thermodynamically conjugated to basic macrovariables $\left\{Q_{j}(\xi, t)\right\}$, what allows a posteriori to generate satisfactory Thermodynamic and Thermo-Hydrodynamic theories. This is accomplished introducing the definition

$$
\varphi_{j}\left(\xi ; t, t^{\prime}\right)=w\left(t, t^{\prime}\right) F_{j}(\xi, t) \quad,
$$

where $w\left(t, t^{\prime}\right)$ is an auxiliary weight function, which, to satisfy the four points just listed immediately above, must have well defined properties which are discussed elsewhere [32], and it is verified that

$$
\Psi(t)=\int_{-\infty}^{t} d t^{\prime} w\left(t, t^{\prime}\right) \phi\left(t^{\prime}\right)
$$

The function $w\left(t, t^{\prime}\right)$ introduces the time-smoothing procedure, and, because of the properties it must have to accomplish its purposes, it is acceptable any kernel that the mathematical theory of convergence of trigonometrical series and transform integrals provides. Kirkwood, Green, Mori and others have chosen what in mathematical parlance is Fejèr (or Cesàro-1) kernel, while Zubarev introduced the one consisting in Abel's kernel for $w$ in Eq. (15) - which apparently appears to be the best choice, either mathematically but mainly physically - that is, taking $w\left(t, t^{\prime}\right)=\varepsilon \exp \left\{\varepsilon\left(t^{\prime}-t\right)\right\}$, where $\varepsilon$ is a positive infinitesimal that goes to zero after the calculation of averages has been performed, and with $t_{o}$ going to minus infinite. Once this choice is introduced in Eq. (12), in Zubarev's approach the nonequilibrium statistical operator, designated by $\rho_{\varepsilon}(t)$, after integration by parts in time, can be written in the form

$$
\rho_{\varepsilon}(t)=\exp \left\{-\hat{S}(t, 0)+\int_{-\infty}^{t} d t^{\prime} e^{\varepsilon\left(t^{\prime}-t\right)} \frac{d}{d t^{\prime}} \hat{S}\left(t^{\prime}, t^{\prime}-t\right)\right\},
$$

where

$$
\hat{S}(t, 0)=-\ln \bar{\rho}(t, 0)=\Phi(t) \hat{1}+\sum_{j} \int d \xi F_{j}(\xi, t) \hat{P}(\xi)
$$

with $\hat{1}$ being the unit operator, and it is introduced the auxiliary operator $\bar{\rho}(t, 0)=\exp \{-\hat{S}(t, 0)\}$, referred-to as an instantaneous quase equilibrium statistical operator, moreover

$\hat{S}\left(t^{\prime}, t^{\prime}-t\right)=\exp \left\{-\frac{1}{i \hbar}\left(t^{\prime}-t\right) \hat{H}\right\} \hat{S}\left(t^{\prime}, 0\right) \exp \left\{\frac{1}{i \hbar}\left(t^{\prime}-t\right) \hat{H}\right\}$.

The operator $\hat{S}(t, 0)$ is designated as the informationalentropy operator, whose relevance and properties are discussed in [45].

In the framework of the nonequilibrium grand-canonical ensemble, namely, when the basic variables are those of Eqs. (4) to (8), we do have that

$$
\bar{\rho}(t, 0)=\exp \{-\hat{S}(t, 0)\} \quad,
$$

where

$$
\begin{gathered}
\hat{S}(t, 0)=\phi(t) \\
+\sum_{r, r^{\prime} \geq 0} \int d^{3} r\left[F_{h}^{[r]}(\mathbf{r}, t) \otimes \hat{I}_{h}^{[r]}(\mathbf{r})+F_{n}^{[r]}(\mathbf{r}, t) \otimes \hat{I}_{n}^{[r]}(\mathbf{r})\right]+ \\
+\sum_{r, r^{\prime} \geq 0} \sum_{p p^{\prime}} \int d^{3} r \int d^{3} r^{r} F_{p p^{\prime}}^{\left[r+r^{\prime}\right]}\left(\mathbf{r}, \mathbf{r}^{\prime}, t\right) \otimes \hat{\mathcal{C}}_{p p^{\prime}}^{\left[r+r^{\prime}\right]}\left(\mathbf{r}, \mathbf{r}^{\prime}\right)
\end{gathered}
$$

where $\otimes$ stands for fully contracted product of tensors, we recall that $r$ and $r^{\prime}$ equal to 0 stands for the densities, and the $F$ 's are the nonequilibrium thermodynamic variables associated to the corresponding observable [13, 46].

Several important points can be stressed in connection with the nonequilibrium statistical operator of Eq. (16). First, the initial condition at time $t_{o} \rightarrow-\infty$, is

$$
\rho_{\varepsilon}\left(t_{o}\right)=\bar{\rho}\left(t_{o}, 0\right)
$$

what implies in a kind of initial Stosszahlanzatz, in the sense that the initial state is defined by the instantaneous generalized canonical-like distribution $\bar{\rho}$, thus ignoring correlations among the basic variables prior to time $t_{o}$. Second, $\rho_{\varepsilon}(t)$ can be separated into two parts [13, 24, 30-33], see also [18], namely,

$$
\rho_{\varepsilon}(t)=\bar{\rho}(t, 0)+\rho_{\varepsilon}^{\prime}(t)
$$

where $\bar{\rho}(t, 0)$ is the instantaneous distribution of Eq. (19). The first one, $\bar{\rho}$, defines an instantaneous, at time $t$, distribution, which describes a "frozen" equilibrium providing at such given time the macroscopic state of the system, and for that reason is sometimes dubbed as the quasi-equilibrium statistical operator. This distribution describes the macrostate of the system in a time interval, around $t$, much smaller than the relaxation times of the basic variables (implying in a "frozen" equilibrium or quasi-equilibrium in such interval). But, of course, for larger time intervals the effect of the dissipational processes comes into action. The dynamics that has led the system to that state at time $t$ from the initial condition of preparation at time $t_{o}$ [cf. Eq. (21)], as well as its continuing dissipative evolution from that state at time $t$ to eventually a 
final full equilibrium, is contained in the fundamental contribution $\rho_{\varepsilon}^{\prime}(t)$. Furthermore, there exists a time-dependent projection operator $\mathcal{P}(t)$ with the property that $[32,33]$

$$
\mathcal{P}(t) \ln \rho_{\varepsilon}(t)=\ln \bar{\rho}(t, 0)
$$

This projection procedure, a generalization of those of Zwanzig (apparently the first to introduce projection techniques in statistical physics [20]), Mori [19], Zubarev and Kalashnikov [27], and Robertson [25], has interesting characteristics. We recall that the formalism involves the macroscopic description of the system in terms of the set of macrovariables $\left\{Q_{j}(\xi, t)\right\}$, which are the average over the nonequilibrium ensemble of the set of dynamical quantities $\left\{\hat{P}_{j}(\xi)\right\}$. The latter are called the "relevant" variables, and we denote the subspace they define as the informational subspace of the space of states of the system. The remaining quantities in the dynamical description of the system, namely, those absent from the informational space associated to the constraints in MaxEnt, are called "irrelevant" variables. The role of the projection operation is to introduce what can be referred to as a coarse-graining procedure, in the sense that it projects the logarithm of the "fine-grained" statistical operator $\rho_{\varepsilon}(t)$ onto the subspace of the "relevant" (informational) variables, this projected part being the logarithm of the auxiliary (or quasi-equilibrium, or "instantaneous frozen", or "coarsegrained") distribution $\bar{\rho}(t, 0)$, and, consequently, the procedure eliminates the "irrelevant" variables, quite in the spirit of the Bayesian-based approach and MaxEnt. The "irrelevant" variables are "hidden" in the contribution $\rho_{\varepsilon}^{\prime}(t)$ to the full distribution $\rho_{\varepsilon}(t)$ of Eq. (22), since it depends on the last term in the exponential of Eq. (16), where the differentiation in time drives $\ln \bar{\rho}$ outside the subspace of "relevant" (informational) variables. We stress that the projection operation is time dependent, such dependence corresponding to the fact that the projection $\mathcal{P}(t)$ is determined by the macroscopic state of the system at the time the projection is performed. Further considerations of this projection procedure will appear in the kinetic and thermodynamics theories based on this informational approach. Moreover, geometrical-topological implications are derived and discussed in detail by Balian et al. [47].

Two further comments are of relevance. First, for a given dynamical quantity $\hat{A}$, its average value in MaxEnt-NESOM, that is, the expected value to be compared with the experimental measure, is given by

$$
\begin{gathered}
\langle\hat{A} \mid t\rangle=\lim _{\varepsilon \rightarrow+0} \operatorname{Tr}\left\{\hat{A} \rho_{\varepsilon}(t)\right\} \\
=\operatorname{Tr}\{\hat{A} \bar{\rho}(t, 0)\}+\lim _{\varepsilon \rightarrow+0} \operatorname{Tr}\left\{\hat{A} \rho_{\varepsilon}^{\prime}(t)\right\},
\end{gathered}
$$

the last equality following after the separation given by Eq. (22) is introduced. This is the said generalization of Kirkwood time-smoothing averaging [16], and we can see that the average value is composed of two contributions: one is the average with the quasi-equilibrium distribution (meaning the contribution of the state at the time $t$ ), plus the contribution arising out of the dynamical behavior of the system (the one that accounts for the past history and future dissipational evolution). Moreover, this operation introduces in the formalism the socalled Bogoliubov's method of quasi-averages $[42,48]$. Bogoliubov's procedure involves a symmetry-breaking process, which is introduced in order to remove degeneracies connected with one or several groups of transformations in the description of the system. According to Eq. (24) the regular average with $\rho_{\varepsilon}(t)$ is followed by the limit of cancelling the ad hoc symmetry-breaking introduced by the presence of the weight function $w$ in Eq. (14) (which is Abel's kernel in Zubarev approach, cf. Eq. (16), and follows for $\varepsilon$ going to +0 ), which imposes a breaking of the time-reversal symmetry in the dynamical description of the system. This is mirrored in the Liouville equation for $\rho_{\varepsilon}(t)$ : Zubarev's nonequilibrium statistical operator does satisfy Liouville equation, but it must be reckoned the fact that the group of its solutions is composed of two subsets, the one corresponding to the retarded solutions and the one corresponding to the advanced solutions. The presence of the weight function $w$ (Abel's kernel in Zubarev's approach) in the time-smoothing or quasiaverage procedure that has been introduced selects the subset of retarded solutions from the total group of solutions of Liouville equation. We call the attention (as Zubarev had; see Appendix in the book of reference [24]) that this has a certain analogy with Gell-Mann and Goldberger [49] procedure in scattering theory, where these authors promote a symmetrybreaking in Bogoliubov's sense in Schroedinger equation, in order to represent the way in which the quantum mechanical state has been prepared during times $-\infty \leq t^{\prime} \leq t$, adopting for the wave function a weighted time-smoothing as the one used in Zubarev's approach to NESEF. More precisely, $\rho_{\varepsilon}(t)$ satisfies a Liouville equation of a form that automatically, via Bogoliubov's procedure, selects the retarded solutions, namely

$$
\frac{\partial}{\partial t} \ln \rho_{\varepsilon}(t)+i \hat{\Lambda}_{\varepsilon}(t) \ln \rho_{\varepsilon}(t)=0 \quad,
$$

where $\hat{\Lambda}_{\varepsilon}$ is the modified Liouville operator

$$
i \hat{\Lambda}_{\varepsilon}(t)=i \hat{\mathcal{L}}+\varepsilon[1-\mathcal{P}(t)]
$$

with $\hat{\mathcal{L}}$ being the regular Liouville operator and $\mathcal{P}(t)$ the projection operator of Eq. (23). Equation (25) is of the form proposed by Ilya Prigogine [50], with $\hat{\Lambda}_{\varepsilon}$ being composed of even and odd parts under time-reversal. Therefore, the timesmoothing procedure introduces a kind of Prigogine's dynamical condition for dissipativity [50, 51].

Using Eq. (23) we can rewrite Eq. (25) in the form

$$
\frac{\partial}{\partial t} \ln \rho_{\varepsilon}(t)+\frac{1}{i \hbar}\left[\ln \rho_{\varepsilon}(t), \hat{H}\right]=-\varepsilon\left[\ln \rho_{\varepsilon}(t)-\ln \bar{\rho}(t, 0)\right],
$$

viz., a regular Liouville equation but with an infinitesimal source, which introduces Bogoliubov's symmetry breaking of time reversal, and is responsible for disregarding the advanced solutions. Equation (27) is then said to have BoltzmannBogoliubov-Prigogine symmetry. Following Zubarev [24], 
Eq. (27) is interpreted as the logarithm of the statistical operator evolving freely under Liouville operator $\hat{\mathcal{L}}$, from an initial condition at time $t_{o}$, and with the system undergoing random transitions, under the influence of the interaction with the surroundings. This is described by a Poisson distribution ( $w$ in the form of Abel's kernel), and the result at time $t$ is obtained by averaging over all $t^{\prime}$ in the interval $\left(t_{o}, t\right)$ [cf. Eq. (12)]. This is the time-smoothing procedure in Kirkwood's sense [cf. Eq. (24)], and therefore, it is introduced information related to the past history in the thermo-hydrodynamic macrostate of the system along its evolution from the initial $t_{o}$.

Two points need be considered here. One is that the initial $t_{o}$ is usually taken in the remote past $\left(t_{o} \rightarrow-\infty\right)$, and the other that the integration in time in the interval $\left(t_{o}, t\right)$ is weighted by the kernel $w\left(t, t^{\prime}\right)$ (Abel's kernel in Zubarev's approach, Fejér's kernel in Kirkwood, Green , Mori approaches; and others are possible). As a consequence the procedure introduces a kind of evanescent history as the system macrostate evolves toward the future from the initial condition at time $t_{o}$ $(\rightarrow-\infty)$. Therefore, the contribution $\rho_{\varepsilon}^{\prime}(t)$ to the full statistical operator, that is, the one describing the dissipative evolution of the state of the system, to be clearly evidenced in the resulting kinetic theory, clearly indicates that it has been introduced a fading memory process. This may be considered as the statistical-mechanical equivalent of the one proposed in phenomenological continuum-mechanical-based Rational Thermodynamics [52, 53]. In Zubarev's approach this fading process occurs in an adiabatic-like form towards the remote past: as time evolves memory decays exponentially with lifetime $\varepsilon^{-1}$.

We may interpret this considering that as time evolves correlations established in the past fad away, and only the most recent ones strongly influence the evolution of the nonequilibrium system; here again is in action Bogoliubov's principle of correlations weakening. This establishes irreversible behavior in the system introducing in a peculiar way a kind of Eddington's time-arrow: Colloquially speaking, we may say that because of its fading memory, the system can only evolve irreversibly towards the future and cannot "remember" how to retrieve the mechanical trajectories that would return it to the past situations (what is attained when neglecting the advance solutions of Liouville equation). In a sense we may say that Boltzmann original ideas are here at work in quite general conditions $[54,55]$, and in its evolution towards the future, once any external perturbating source is switched off, the system tends to a final state of equilibrium irrespective of the nonequilibrium initial condition of preparation.

Alvarez-Romero and Garcia-Colin [34] has presented an interesting alternative approach to the derivation of Zubarev's form of MaxEnt-NESEF, which however differs from ours in the interpretation of the time-smoothing procedure, which they take as implying the connection of an adiabatic perturbation for $t^{\prime}>t_{o}$ (we think that these authors mean adiabatic switch on of the interactions in $H^{\prime}$ responsible for the dissipative processes), instead of implying in a fading-memory interpretation. We need notice that both are interpretations which we feel are equally satisfactory and may be equivalent, but we side with the point of view of irreversible behavior follow- ing from - in Boltzmann-Bogoliubov-Prigogine's sense - adiabatic decorrelation of processes in the past. This is the fadingmemory phenomenon, introduced in Zubarev's approach as a result of the postulated Poissonian random processes (on the basis that no real system can be wholly isolated), as already discussed. This interpretation aside, we agree with the authors in Ref. [34], in that the method provides adequate convergence properties (ensured by Abel's kernel in Zubarev' approach) for the equations of evolution of the system. These properly describe the irreversible processes unfolding in the media, with an evolution from a specific initial condition of preparation of the system and, after remotion of all external constraints - except thermal and particle reservoirs - tending to the final grand-canonical equilibrium distribution.

Moreover, the convergence imposed by Abel's kernel in Zubarev's approach appears as the most appropriate, not only for the practical mathematical advantages in the calculation it provides, but mostly important, by the attached physical meaning associated to the proposed adiabatic decoupling of correlations which surface in the transport equations in the accompanying MaxEnt-NESEF kinetic theory [56]. In fact, on the one hand this kinetic theory produces, when restrictions are applied on the general theory, the expected collision operators (as those derived in other kinetic theories) introducing, after the time integration in the interval $\left(t_{o}, t\right)$ has been done, the expected terms of energy renormalization and energy conservation in the collision events. Furthermore, as pointed out by Zubarev [24], Abel's kernel ensures the convergence of the integrals in the calculation of the transport coefficients, which in some cases show divergences when, instead, Fejèr kernel is used (as in Green, Mori, etc. approaches). The procedure also appears as having certain analogies with the so-called repeated randomness assumptions $[57,58]$ as discussed by del Rio and Garcia-Colin [59].

We need now to consider the construction of a MaxEntNESEF-based Nonlinear Kinetic Theory, that is, the transport (evolution) equations for the basic set of macrovariables that describe the irreversible evolution of the macrostate of the system. They are, in principle, straightforwardly derived, consisting in Heisenberg equations of motion for the corresponding basic dynamical variables (mechanical observables) or Hamilton equations in the classical case, averaged over the nonequilibrium ensemble, namely

$$
\frac{\partial}{\partial t} Q_{j}(\xi, t)=\operatorname{Tr}\left\{\frac{1}{i \hbar}\left[\hat{P}_{j}(\xi), \hat{H}\right] \rho_{\varepsilon}(t)\right\} \quad .
$$

Using the separation of the Hamiltonian as given by $\hat{H}=$ $\hat{H}_{o}+\hat{H}^{\prime}$, where $\hat{H}_{o}$ is the kinetic energy and $\hat{H}^{\prime}$ contains the interaction and the separation of the statistical operator as given by Eq. (22), it follows that Eq. (28) can be written in the form $[56,60]$

$$
\frac{\partial}{\partial t} Q_{j}(\xi, t)=J_{j}^{(0)}(\xi, t)+J_{j}^{(1)}(\xi, t)+g_{j}(\xi, t),
$$

where on the right-hand side are present the contributions

$$
J_{j}^{(0)}(\xi, t)=\operatorname{Tr}\left\{\frac{1}{i \hbar}\left[\hat{P}(\xi), \hat{H}_{o}\right] \bar{\rho}(t, 0)\right\},
$$




$$
\begin{gathered}
J_{j}^{(1)}(\xi, t)=\operatorname{Tr}\left\{\frac{1}{i \hbar}\left[\hat{P}(\xi), \hat{H}^{\prime}\right] \bar{\rho}(t, 0)\right\} \\
\mathcal{g}_{j}(\xi, t)=\operatorname{Tr}\left\{\frac{1}{i \hbar}\left[\hat{P}(\xi), \hat{H}^{\prime}\right] \rho_{\varepsilon}^{\prime}(t)\right\}
\end{gathered} .
$$

As shown elsewhere $[32,56,60]$ this Eq. (29) can be considered as a far-reaching generalization of Mori's equations $[19,61]$. It also contains a large generalization of Boltzmann's transport theory, with the original Boltzmann equation for the one-particle distribution retrieved under stringent asymptotic limiting conditions; details and discussions are given in Refs. [33] and [62].

In this Eq. (29), in most cases of interest the contribution $J^{(1)}$ is null because of symmetry properties of the interactions in $\hat{H}^{\prime}$, and the term $J^{(0)}$ provides a conserving part consisting in the divergence of the flux of quantity $Q_{j}(\xi, t)[63,64]$. The last term, i.e. the one of Eq. (32), is the collision integral responsible for relaxation processes, which, evidently, cancels if $\hat{H}^{\prime}$ or $\rho_{\varepsilon}^{\prime}$ is null, what clearly indicates that dissipative phenomena are described by these contributions to the Hamiltonian, and to the statistical operator in Eq. (22), respectively. Hence, as already anticipated, dissipation is not present in the instantaneous quasi-equilibrium operator $\bar{\rho}(t, 0)$ of Eq. (19), but in the nonequilibrium operator containing the history and time-smoothing characteristic of $\rho_{\varepsilon}^{\prime}(t)$ of Eqs. (16) and (22). We notice that if $\hat{H}^{\prime}$ is null, so is $\rho_{\varepsilon}^{\prime}(t)$, when $\hat{H}_{o}$ coincides with the whole Hamiltonian corresponding to a full equilibrium condition.

The collision integral of Eq. (32) requires an, in general, quite difficult, and practically unmanageable, mathematical handling. But for practical use, it can be reformulated in the form of an infinite series of partial collision integrals in the form

$$
g_{j}(\xi, t)=\sum_{n=2}^{\infty} \Omega_{j}^{(n)}(\xi, t)
$$

where quantities $\Omega^{(n)}$ for $n=2,3, \ldots$ can be interpreted as describing two-particle, three-particle, etc., collisional processes. These partial collision integrals, and then the transport equation (29), are highly nonlinear, with complete details given in Refs. [56, 60].

An interesting limiting case is the Markovian approximation to Eq. (29), consisting into retaining in the collision integral of Eq. (33) the interaction $\hat{H}^{\prime}$ strictly up to second order (limit of weak interactions) $[13,60,65]$ to obtain for a density $Q_{j}(\xi, t)$ the equation $[13,22,63,64]$

$$
\frac{\partial}{\partial t} Q_{j}(\xi, t)=J_{j}^{(0)}(\xi, t)+J_{j}^{(2)}(\xi, t)
$$

where

$$
J_{j}^{(2)}(\xi, t)=\int_{-\infty}^{t} d t^{\prime} e^{\varepsilon\left(t^{\prime}-t\right)} \operatorname{Tr}\left\{\left[\hat{H}^{\prime}\left(t^{\prime}-t\right)_{0},\left[\hat{H}^{\prime}, \hat{P}_{j}(\xi)\right]\right] \bar{\rho}(t, 0)\right\}
$$

once $J_{j}^{(1)}$ is taken as null, and subindex nought indicates mechanical evolution under $\hat{H}_{o}$ alone (interaction representation).

Finally, an additional step is the construction of the all important MaxEnt-NESEF response function theory for systems arbitrarily away from equilibrium, to connect theory with observation and measurement in the experimental procedure: see for example [66-81] and Chapter 6 in the book of Ref. [13]. We simply notice that as in the traditional response function theory around equilibrium $[82,83]$, the response of the system away from equilibrium to an external probe is expressed in terms of correlation functions but defined over the nonequilibrium ensemble. Moreover, also in analogy with the case of systems in equilibrium it is possible to construct a double time nonequilibrium thermodynamic Green function formalism [84-87].

In this way, through the realization of the basic steps we have described, a nonequilibrium statistical ensemble formalism - the MaxEnt-NESEF - can be built. Finally, it can be noticed that it is a formalism in the Theory of Irreversible Processes developed along a traditional line (Gibbs' ensemble algorithm for nonequilibrium systems), which is an alternative to the extensively used Nonequilibrium Molecular Dyanmics.

\section{Acknowledgments}

We acknowledge financial support to our Group provided in different opportunities by the São Paulo State Research Foundation (FAPESP), the Brazilian National Research Council (CNPq), the Ministry of Planning (Finep), the Ministry of Education (CAPES), Unicamp Foundation (FAEP), IBM Brasil, and the John Simon Guggenheim Memorial Foundation (New York, USA).
[1] N. Oreskes, H. Shrader-Frechette, and K. Beltz, Science 263, 641 (1994).

[2] O. Penrose, Rep. Prog. Phys. 42, 1938 (1979).

[3] R. Kubo, Prog. Theor. Phys. (Japan) Suppl. 64, 1 (1978).

[4] L. Sklar, Physics and Chance: Philosophical Issues in the Foundations of Statistical Mechanics (Cambridge Univ. Press, Cambridge, UK, 1993).

[5] H. G. B. Casimir, Rev. Mod. Phys. 17, 343 (1945).
[6] J. Semura, Am. J. Phys. 64, 526 (1996).

[7] E. T. Jaynes, in Frontiers of Nonequilibrium Statistical Physics, edited by G. T. Moore and M. O. Scully (Plenum, New York, USA, 1986), pp. 33-55.

[8] E. T. Jaynes, in The Maximum Entropy Formalism, edited by M. Tribus and R. D. Levine (MIT Press, Cambridge, MA, USA, 1978), pp. 15-118.

[9] E. T. Jaynes, in Complex Systems: Operational Approaches, 
edited by H. Haken (Springer, Berlin, Germany, 1985).

[10] E. T. Jaynes, in E. T. Jaynes Papers on Probability, Statistics, and Statistical Physics, edited by R. D. Rosenkrantz (ReidelKluwer Academic, Dordrecht, The Netherlands, 1983).

[11] E. T. Jaynes, Proc. IEEE 70, 939 (1982).

[12] E. T. Jaynes, in Maximum Entropy and Bayesian Methods, edited by J. Skilling (Kluwer Academic, Dordrecht, The Netherlands, 1989), pp. 1-27.

[13] R. Luzzi, A. R. Vasconcellos, and J. G. Ramos, Predictive Statistical Mechanics : A Nonequilibrium Ensemble Formalism (Kluwer Academic, Dordrecht, The Netherlands, 2002).

[14] R. Luzzi, A. R. Vasconcellos, and J. G. Ramos, Theory of Irreversible Processes, IFGW-Unicamp Internal Report (2005) and future publication.

[15] R. Zwanzig, in Perspectives in Statistical Physics, edited by H. J. Ravechè (North Holland, Amsterdam, The Netherlands, 1981), pp. 123-124.

[16] J. G. Kirkwood, J. Chem. Phys. 14, 180 (1946).

[17] M. S. Green, J. Chem. Phys. 20, 1281 (1952).

[18] H. Mori, I. Oppenheim, and J. Ross, in Studies in Statistical Mechanics I, edited by J. de Boer and G. E. Uhlenbeck (North Holland, Amsterdam, The Netherlands, 1962), pp. 217-298.

[19] H. Mori, Progr. Theor. Phys. (Japan) 33, 423 (1965).

[20] R. Zwanzig, in Lectures in Theoretical Physics, edited by W. E. Brittin, B. W. Downs, and J. Downs (Wiley-Interscience, New York, USA, 1961).

[21] J. A. McLennan, Advances in Chemical Physics (Academic, New York, USA, 1963), Vol. 5, pp. 261-317.

[22] S. V. Peletminskii and A. A. Yatsenko, Soviet Phys. JETP 26, 773 (1968), [zh. Ekps. Teor. Fiz. 53, 1327 (1967)].

[23] A. I. Akhiezer and S. V. Peletminskii, Methods of Statistical Physics (Pergamon, Oxford, UK, 1981).

[24] D. N. Zubarev, Nonequilibrium Statistical Thermodynamics (Consultants Bureau, New York, USA, 1974), [Neravnovesnaia Statisticheskaia Termodinamika (Izd. Nauka, Moscow, Russia, 1971)].

[25] B. Robertson, Phys. Rev. 144, 151 (1966).

[26] H. Grabert, Projection Operators Techniques in Nonequilibrium Statistics (Springer, Berlin, Germany, 1981).

[27] D. N. Zubarev and V. P. Kalashnikov, Physica 56, 345 (1971).

[28] A. Salams, V. Vladimorov, and A. Logunov, Theor. Math. Phys. 92, 817 (1993), [Teor. Mat. Fiz. 92, 179 (1992)].

[29] N. S. Krylov, Works on the Foundations of Statistical Mechanics (Princeton Univ. Press, Princeton, USA, 1979).

[30] D. N. Zubarev, V. N. Morozov, and G. Röpke, Statistical Mechanics of Nonequilibrium Processes: Basic Concepts, Kinetic Theory (Akademie Wiley-VHC, Berlin, Germany, 1996).

[31] D. N. Zubarev and V. P. Kalashnikov, Theor. Math. Phys. 1, 108 (1970), [Teor. Mat. Fiz. 1, 137 - (1969)].

[32] R. Luzzi and A. R. Vasconcellos, Fortschr. Phys./Prog. Phys. 38, 887 (1990).

[33] J. G. Ramos, A. R. Vasconcellos, and R. Luzzi, Fortschr. Phys./Prog. Phys. 43, 265 (1995).

[34] J. T. Alvarez-Romero and L. S. Garcia-Colin, Physica A 232, 207 (1996).

[35] R. Baierlein, Am. J. Phys. 63, 108 (1995).

[36] H. Jeffreys, Scientific Inference (Cambridge Univ. Press, Cambridge, UK, 1973).

[37] H. Jeffreys, Probability Theory (Clarendon, Oxford, UK, 1961).

[38] P. W. Anderson, Phys. Today 45 (1), 9 (1992).

[39] A. J. Garret, Contemp. Phys. 33, 271 (1992).

[40] C. E. Shannon and W. Weaver, The Mathematical Theory of Communication. (Univ. Illinois Press, Urbana, USA, 1948).

[41] L. Brillouin, Science and Information Theory (Academic Press,
New York, USA, 1962).

[42] N. N. Bogoliubov, in Studies in Statistical Mechanics I, edited by J. de Boer and G. E. Uhlenbeck (North Holland, Amsterdam, The Netherlands, 1962).

[43] U. Fano, Rev. Mod. Phys. 29, 74 (1957).

[44] R. Peierls, Lecture Notes in Physics (Springer, Berlin, Germany, 1974), Vol. 31.

[45] S. A. Hassan, A. R. Vasconcellos, and R. Luzzi, Physica A 262, 359 (1999).

[46] R. Luzzi, A. R. Vasconcellos, and J. G. Ramos, Statistical Foundations of Irreversible Thermodynamics (TeubnerBertelsmannSpringer, Stuttgart, Germany, 2000).

[47] R. Balian, Y. Alhassid, and H. Reinhardt, Phys. Rep. 131, 1 (1986).

[48] N. Bogoliubov Jr, A Method for Studying Model Hamiltonians (Pergamon, Oxford, UK, 1972).

[49] M. Gell-Mann and M. L. Goldberger, Phys. Rev. 91, 398 (1953).

[50] I. Prigogine, From Being to Becoming (Freeman, San Francisco, USA, 1980).

[51] I. Prigogine, Nature 246, 67 (1975).

[52] C. Truesdell, Rational Thermodynamics (McGraw-Hill, New York, USA, 1985), [second enlarged edition (Springer, Berlin, Germany, 1988)].

[53] J. Meixner, in Irreversible Processes of Continuum Mechanics, edited by H. Parkus and L. Sedov (Springer, Wien, Austria, 1968).

[54] J. L. Lebowitz, Phys. Today 46 (9), 32 (1993), see also Letters Section in Physics Today 47 (11), pp. 13-15 and 115-116 (1994).

[55] J. L. Lebowitz, Phys. Today (Letters) 47 (11), 115 (1994).

[56] L. Lauck, A. R. Vasconcellos, and R. Luzzi, Physica A 168, 789 (1990).

[57] N. G. V. Kampen, in Perspectives in Statistical Physics, edited by H. Ravechè (North Holland, Amsterdam, The Netherlands, 1981), p. 91.

[58] N. V. Kampen, in Fundamental Problems in Statistical Mechanics, edited by E. Cohen (North Holland, Amsterdan, The Netherlands, 1962), p. 173.

[59] J. D. Rio and L. G. Colin, Phys. Rev. E 54, 950 (1996).

[60] J. Madureira, A. Vasconcellos, R. Luzzi, and L. Lauck, Phys. Rev. E 57, 3637 (1998).

[61] D. Forster, Hydrodynamic Fluctuations, Broken Symmetry, and Correlation Functions (Benjamin, Readings, USA, 1975).

[62] J. G. Ramos, A. R. Vasconcellos, and R. Luzzi, Physica A 284, 140 (2000).

[63] J. Madureira, A. Vasconcellos, and R. Luzzi, J. Chem. Phys. 109, 2099 (1998).

[64] S. V. Peletminskii and A. I. Sokolovskii, Theor. Math. Phys.(USSR) 18, 85 (1974).

[65] D. Zubarev, in reference [24], see Chapter IV, Section 22 .

[66] D. K. Ferry, H. L. Grubin, and G. J. Giafrate, in Semiconductors Probed by Ultrafast Laser Spectroscopy, edited by R. R. Alfano (Academic Press, New York, USA, 1984), Vol. 1, pp. 413-447.

[67] D. Y. Xing, P. Hiu, and C. S. Ting, Phys. Rev. B 35, 6379 (1987).

[68] V. N. Freire, A. R. Vasconcellos, and R. Luzzi, Phys. Rev. B 39, 13264 (1988).

[69] R. Luzzi and L. C. Miranda, Physics Reports Reprint Books Series (North Hollland, Amsterdam, The Netherlands, 1978), Vol. 3, pp. 423-453.

[70] R. Luzzi and A. R. Vasconcellos, in Semiconductor Processes Probed by Ultrafast Laser Spectroscopy, edited by R. R. Alfano (Academic, New York, USA, 1984), Vol. 1, pp. 135-169. 
[71] R. Luzzi, in High Excitation and Short Pulse Phenomena, edited by M. H. Pilkuhn (North Holland, Amsterdam, The Netherlands, 1985), pp. 318-332.

[72] A. C. Algarte and R. Luzzi, Phys. Rev. B 27, 7563 (1983).

[73] A. C. Algarte, Phys. Rev. B 38, 2162 (1988).

[74] A. R. Vasconcellos and R. Luzzi, Complexity 2, 42 (1997).

[75] A. C. Algarte, A. R. Vasconcellos, and R. Luzzi, Braz. J. Phys. 26, 543 (1996)

[76] A. R. Vasconcellos, R. Luzzi, D. Jou, and J. Casas-Vázquez, J. Chem. Phys. 107, 7383 (1998).

[77] A. C. Algarte, A. R. Vasconcellos, and R. Luzzi, Phys. Stat. Sol. (b) 173, 487 (1992)

[78] A. C. Algarte, Phys. Rev. B 43, 2408 (1991).

[79] A. C. Algarte, A. R. Vasconcellos, and R. Luzzi, Solid State Commun 87, 299 (1993).

[80] A. R. Vasconcellos, A. C. Algarte, and R. Luzzi, Phys. Rev. B 48, 10873 (1993).

[81] R. Luzzi, A. R. Vasconcellos, J. Casas-Vázquez, and D. Jou,
Physica A 248, 111 (1997).

[82] P. C. Martin, in Many-Body Physics, edited by C. D. Witt and R. Balian (Gordon and Breach, New York, USA, 1968), pp. 37136.

[83] H. J. Kreuzer, Nonequilibrium Thermodynamics and its Statistical Foundations (Clarendon, Oxford, UK, 1981).

[84] R. Luzzi and A. R. Vasconcellos, J. Stat. Phys. 23, 539 (1980).

[85] A. R. Vasconcellos, J. G. Ramos, M. V. Mesquita, and R. Luzzi, Response Function Theory in a Nonequilibrium Ensemble Formalism, IFGW-Unicamp Internal Report (2005), and future publication.

[86] A. R. Vasconcellos, J. G. Ramos, M. V. Mesquita, and R. Luzzi, Scattering Theory in a Nonequilibrium Ensemble Formalism, IFGW-Unicamp Internal Report (2005), and future publication.

[87] V. P. Kalashnikov, Theor. Math. Phys. 35, 362 (1978), [teor. Mat. Fiz. 35, 127 (1978)]. 